\title{
A ABDUÇÃO DOS ARTELHOS E O SINAL DO LEQUE
}

(BABINSKI, 1903)

\author{
RICARDO DE OLIVEIRA-SOUZA *, WAGNER MARTIGNONI DE FIGUEIREDO* *
}

RESUMO - A abdução dos artelhos meriores ("sinal do leque") coricomitante à adoção da atitude sentada a partir do decúbito (movimento associado) ou em resposta à excitação plantar externa (resposta reflexa) foi formalmente descrita por Babinski em 1903 e contrastada com a dorsiflexão do hálux em termos dos seus respectivos valores de localização. No presente ensaio traduzimos as duas comunicaçōes em que a abdução dos artelhos recebeu sua caracterização semiológica final e enfatizamos a distinção entre o fenômeno da abdução e a extensāo reflexa dos artelhos, particularmente do grande. Aduzimos, ainda, evidências de que, na literatura anátomo-clínica e neurofisiológica dos anos subsequentes, 0 assim chamado "sinal de Babinski" e respostas coadjuvantes continuam gerando imprecisões conceituais, descrições inadequadas e investigaçōes imperfeitas.

PALAVRAS-CHAVE: sinal de Babinski, sinal do leque, reflexo, síndrome piramidal.

The abduction of the toes and the fan sign (Babinski, 1903)

ABSTRACT - The abduction of the lesser toes which may be observed as an associated movement or as a reflex response to stroking of the lateral plantar surface of the foot (the fan sign) was formally described in 1903 by Babinski, who compared it to the dorsiflexion of the great toe in terms of their respective values as localizing signs. In the present essay we translated into portuguese and review the two communications in which the characterization of the abduction of the toes was made, emphasizing the distinction between this phenomenon and the reflex dorsiflexion of the tocs, particularly the great toc. Evidence is adduced from the clinical and physiological literature of the succeeding years that shows that the so-called "sign of Babinski" is still surrounded by mists of inadequate clinico-anatomic concepts whence the wealth of incrogruous pathophysiological interpretations which abound in the literature of the past decades.

KEY WORDS: sign of Babinski, fan sign, reflex, pyramidal syndrome.

A estimulação móvel, lenta e firme (fr.: chatouillement, ing.: stroking) do arco plantar de uma pessoa normal deitada, dá lugar a tênue flexão dos dedos, habitualmente associada à retirada da perna, que varia de um esboço de flexão ao afastamento de todo membro da fonte de estimulação ("reflexo da tríplice flexão" ${ }^{19}$ ). Em determinados pacientes, a fração mais distal da resposta se modifica pela conversão da flexão do hálux em extensão, cuja amplitude aumenta conforme o estímulo desliza pelo arco plantar no sentido póstero-anterior. Com frequência, os outros dedos também se estendem (dorsifletem), mas esse movimento conjunto, embora incluído na descrição original do "fenômeno dos artelhos", foi logo em seguida descartado a favor da extensão pura do hálux ${ }^{21}$. Năo obstante, apesar da valorização da resposta do hálux como fundamental, os movimentos dos artelhos menores têm sido considerados parte integrante do "sinal de Babinski" por autores influentes, o que contribuiu

*Professor Assistente, Serviço de Clínica Médica C (Prof. Omar da Rosa Santos), Hospital Universitário Gaffrée e Guinle, Universidade do Rio de Janeiro (HUGG-UNI-RIO); **Professor Adjunto, Serviço de Clínica Médica C (Prof. Omar da Rosa Santos), HUGG-UNI-RIO. Aceite: 15-abril-1996.

Dr. Ricado de Oliveira Souza - Rua General Belford 226 - 20961-000 Rio de Janeiro RJ - Brasil. 
para confundir a matéria e ameaçar a consistência descritiva e conceitual do próprio sinal. No presente estudo, procuramos demonstrar a tese de que o movimento dos artelhos menores jamais representou qualquer problema para o espírito inquisitivo de Babinski. Para atender a este objetivo, traduzimos na íntegra, os dois textos em que abordou os movimentos dos artelhos, especificamente a abdução. Ambos foram publicados como comunicaçnes sucintas no volume de 1903 da Revue Neurologique, cinco anos depois do trabalho fundamental sobre a inversão do reflexo plantar ${ }^{1}$. Poucas vezes, depois disto, Babinski retornaria ao tema ${ }^{5}$. Ressaltamos, dentre outros aspectos, a escolha do termo "perturbação", no lugar de, por exemplo, "lesāo" ou "disfunçāo", para descrever o acometimento do sistema piramidal.

\section{De l'abduction des orteils ${ }^{3}$}

L'excitation de la plante du pied provoque parfois, entre autres mouvements réflexes, une abduction plus ou moins marquée d'un ou de plusieurs orteils qui a déjà été incidemment signalée par certains auteurs qu'ils y aient attaché une valeur sêmiologique quelconque.

Mon attention a été attirée aussi, depuis assez longtemps, sur ce phénomène que $j$ 'ai observé à l'état pathologique. Mais chez les sujets sains il est rare, et quand il existe il est peu prononcé, tandis que chez les malades atteints d'une perturbation du système pyramidal il est bien plus commun, sans l'être toutefois autant que l'extension du gros orteil, et il est parfois très marqué. Il m'a paru sourtout très développé dans les paralysies spasmodiques congénitales accompagnées d'athétose qui consiste d'ailleurs, en partie, en des mouvements d'abduction des orteils. J'ajoute à cela que chez le nouveau-né, dont le système pyramidal n'est pas encore constitué, le chatouillement de la plante du pied donne lieu généralement à une abduction des orteils en même temps qu'à une extension du gros orteil.

Ce fait seul qu'il peut exister à l'état normal m'empêche d'attribuer à ce phénomène l'importance fondamentale qui appartient à l'extension du gros orteil, caractéristique d'une perturbation du système pyramidal; néanmoins, quand il est très accentué, il me parait avoir une certaine signification. Récemment, dans un cas de paraplégie crurale consécutive à un traumatisme ayant motivé une expertise médico-légale, l'absence de tout signe objectif classique d'affection organique du système nerveux avait conduit les médecins chargés de l'examen à émettre l'avis qu'il s'agissait d'hystérie ou de

\section{Sobre a abdução dos artelhos}

A excitaçāo da planta do pé provoca, às vezes, dentre outros movimentos reflexos, uma abdução mais ou menos evidente de um ou mais artelhos, já incidentalmente assinalada por outros autores, que the atribuiram algum valor semiológico.

Também minha atenção se voltou, há muito, para o fenômeno que tenho observado tanto no estado normal quanto patológico. Nos indivíduos sadios, todavia, é raro e, quando existe, pouco pronunciado, ao passo que, nos doentes atingidos por uma perturbação do sistema piramidal, bem mais comum, ainda que não tanto quanto a extensão do grande artelho mas, às vezes, bem nítido. Pareceu-me sobretudo bem desenvolvido nas paralisias espasmódicas congênitas acompanhadas de atetose, que consiste, em parte, de movimentos de abdução dos artelhos. Acrescento que, no recém-nascido, cujo sistema piramidal ainda não está constituído, a excitação da planta do pé cede lugar, geralmente, à abdução dos artelhos concomitante à extensāo do grande artelho.

O fato singular de que possa existir no estado normal me impede de atribuir ao fenômeno importância tão fundamental quanto à extensão do grande artelho, característica de perturbação do sistema piramidal; entretanto, quando muito acentuado, parece-me dotado de certa significação. Recentemente, em um caso de paraplegia crural consecutiva a traumatismo que motivou perícia médico-legal, a ausência de qualquer sinal objetivo clássico de afecção orgânica do sistema nervoso levou os médicos encarregados do exame a emitir parecer de que se tratava de histeria ou simulação; tendo observado, no paciente, abdução muito nítida dos 
simulation; ayant observé chez ce malade une abduction très nette des orteils, j'ai émis une opinion contraire et, environ trois semaines après ma première consultation, un deuxième examen me permettait de constater une extension des orteils qui avait fait défault jusque-là et qui confirmait ma manière de voir.

J'estime donc que l'abduction des orteils, dans les conditions que je viens de spécifier, constitue un signe de probabilité en faveur d'une perturbation du système pyramidal, qui peut être précieux dans certaines cas doutex. artelhos, emiti opinião contrária e, cerca de três semanas depois da minha primeira consulta, um segundo exame permitiu-me constatar a extensão dos artelhos até então ausente e confirmar meu ponto de vista.

Acredito, assim, que a abdução dos artelhos, nas condições que acabo de especificar, constitui sinal de probabilidade de perturbação do sistema piramidal, eventualmente preciosa em certos casos duvidosos.

Babinski ilustrou seu argumento com uma fotografia notável (Figura). No texto complementar, transcrito adiante, assinalou que a resposta abdutora podia ser reproduzida por dois caminhos distintos: pela excitação da planta do pé (a resposta reflexa, reportada acima) ou como movimento associado à adoção da posição sentada a partir do decúbito (não reflexo). Deve-se enfatizar, ainda, que a $a b d u c ̧ \tilde{o} o$ dos artelhos - o "sinal do leque" -diferia em aspectos essenciais da extensão que acompanha a resposta patológica do hálux, descrita originalmente em 1896 no estudo sobre o "fenômeno dos artelhos" e que integra nosso cotidiano de beira-de-leito ${ }^{21}$.

\section{De l'abduction des orteils (Signe de l'éventeil) ${ }^{4}$}

J'ai montré dans une précédente communication que l'abduction réflexe provoqué par une excitation de la plante du pied constitue, quand elle est bien marquée, une présomption de perturbation du système pyramidal.

\section{Sobre a abdução dos artelhos (Sinal de leque)}

Conforme demonstrei em comunicação precedente, a abdução reflexa provocada pela excitação da planta do pé, constitui, quando bem marcada, presunção de perturbação do sistema piramidal.

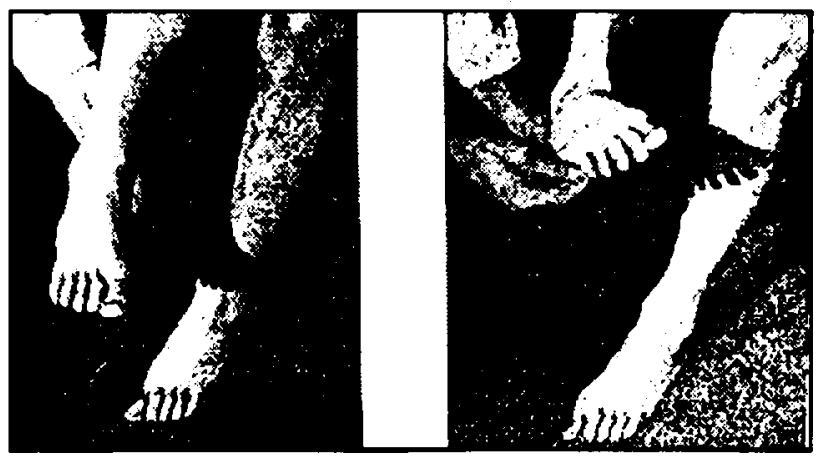

Figura. Ilustração original da abdução dos artelhos'. À esquerda, antes da pesquisa do reflexo. À direita, a resposta típica. Observe-se a projeçāo da sombra da abertura dos artelhos na perna normal do paciente. Reprodução da figura autorizada por Révue Neurologique (Dr. J.M. Léger, Editor Chefe, 25-março-1996), pelo que os autores deste artigo agradecem. 
Des faits plus récemment observés me conduisent à admettre que cette perturbation peut occasioner aussi une abduction associée des orteils. Pour constater ce phénomène, voici dans quelles conditions il faut se placer: le sujet en observation doit se coucher sur le dos; puis, après avoir croisé les bras sur la poitrine, exécuter des mouvements alternatifs de flexion et d'extension du tronc sur le bassin, comme pour la recherche $\mathrm{du}$ "mouvement combiné de flexion du tronc et de la cuisse". Pendant l'éxecution de ces actes on voit les orteils s'écarter les uns des autres.

L'abduction associée des orteils me parait avoir une signification clinique de même ordre que l'abduction réflexe; mais il faut remarquer que si ces deux espèces de mouvements coexistent parfois, ils peuvent aussi exister l'un en absence de l'autre.

L'abduction associée est un phénomène assez rare qui m'a semblé plus commun dans l'hémiplegie infantile que dans celle de l'adulte, plus fréquente dans l'hémiparesie que dans l'hémiplegie.

Notre collègue $M$. Dupré propose de donner à l'abduction des orteils, qu'elle soit d'origine réflexe, ou qu'elle se manifeste comme un mouvement associé, la dénomation de signe de l'eventeil. C'est une expression imagée qui mérite d'être rétenue; mais, comme il peut y avoir intéret à spécifier les conditions dans lesquelles ce signe se produit, il est peut-être préférable de se contenter des termes abduction des orteils, auxquels on ajoutera, suivant les circonstances, les mots réflexe, ou associée, ou réflexe et associée.

J'ai observé abduction associée des orteils du côté droit chez une malade atteinte d'un spasme fonctionnel du membre supérieur droit qui se manifestait en particulier par une crampe des écrivains; cette femme présentait en même temps à droite le phénomène de flexion combinée de la cuisse et du tronc. Ce fait me suggère une idée analogue à celle que j'ai émise sur la pathogénie du torticolis dit mental, à savoir que le spasme fonctionnel est peut-être, du moins dans certains cas, sous la dépendence d'une perturbation du système pyramidal; mais c'est n'est encore qu'une hypothèse qui a besoin de vérification.
Fatos de observação mais recente levamme a admitir que esta perturbação pode ocasionar, também, uma abdução associada dos artelhos. Para constatar o fenômeno, eis as condiçōes necessárias: o sujeito sob observação deve se deitar de costas; em seguida, após cruzar os braços sobre o peito, executar movimentos alternantes de flexão e extensão do tronco sobre a bacia, como na pesquisa do "movimento combinado de flexão do tronco sobre a coxa". Durante e execução desses atos, observamos os artelhos se afastarem uns dos outros.

A abdução associada dos artelhos pareceme dotada do mesmo significado clínico que a abdução reflexa; mas deve-se assinalar que estas duas espécies de movimentos às vezes coexistam, podem, também, existir independentemente uma da outra.

A abdução associada é fenômeno muito raro, a meu ver mais comum na hemiplegia da criança do que na do adulto, mais frequente na hemiparesia do que na hemiplegia.

Nosso colega Dupré propôs denominar a abdução dos artelhos, seja de origem reflexa, seja manifestação de um movimento associado, como o sinal do leque. Trata-se de expressão metafórica que merece ser adotada; todavia, como pode ser de interesse especificar as condiçōes nas quais o sinal é produzido, talvez seja preferível limitarmo-nos aos termos abdução dos artelhos, aos quais se acrescentará, segundo as circunstâncias, as palavras reflexa, ou associada, ou reflexa e associada.

Observei a abduçāo associada dos artelhos do lado direito de uma paciente acometida de espasmo funcional do membro superior direito, que se manifestava, em particular, por cãimbra dos escrivães; apresentava ao mesmo tempo, à direita, o fenômeno da flexão combinada da coxa e do tronco. Tal fato me sugere idéia análoga à que pronunciei sobre a patogênese do torcicolo dito mental, ou seja, que o espasmo funcional, ao menos em certos casos, talvez se encontre na dependência de uma perturbação do sistema piramidal; isto, contudo, năo passa de uma hipótese que necessita verificação. 


\section{DISCUSSÄO}

Os textos acima demonstram que a consulta às origens da neurologia é dotada de utilidade prática legítima, antes de se constituir em simples exercício acadêmico. Seu estudo não só nos protege da inevitável erosão conceitual do transcorrer dos anos, como instrui sobre técnicas e conceitos clínicos que, mesmo hoje em dia, dificilmente poderiam ser elaborados.

A importância semiológica dos dedos dos pés se deve à variedade de movimentos potencialmente significativos que podem efetuar, tanto em condições normais como em pessoas doentes. De todos, a extensão reflexa do hálux em resposta à excitação plantar é o mais relevante, dada a sua singularidade como indicador de afecção do sistema nervoso ${ }^{15}$. O fato de que os contemporâneos de Babinski detinham exata noção do que se constituía o sinal mostra que as imprecisð̄es não eram necessárias. Para Dejerine, por exemplo", "em casos de lesão ou irritação do feixe piramidal, o reflexo plantar normal, em flexão, é substituido pelo movimeto de extensão (flexão dorsal) do grande artelho. Este é (...) o sinal de Babisnki"(itálico no original). Infelizmente, para muitos, a resposta dos outros dedos sempre confundiu a questāo, pois recebeu o mesmo valor semiologico da extensāo do grande artelho'. Em poucos anos, tal ambiguidade foi assimilada pela fisiologia experimental que, conforme exemplificado abaixo, também parece ter negligenciado os textos fundamentais.

No âmbito clínico-semiológico, apenas para dar alguns exemplos, em reverenciado Tratado de Medicina do ínicio do século, Bouchard e Brissaud7 ensinavam que "na hemiplegia orgânica, a mesma excitação provoca no lado paralisado, a extensão dorsal dos artelhos (sinal de Babinski), particularmente do grande". Em um clássico sobre o exame neurológico, Bickerstaff ${ }^{6}$ afirnou que "a resposta de Babinski consiste na extensão do hálux na articulação metatarso-falangeana (...) acompanhada de abertura em leque e dorsiflexão dos otros dedos". Cossa, autor de uma das primeiras obras de fisiopatologia neurológica ${ }^{10}$, assinalou que "à excitação da planta do pé, no caso de lesăo piramidal, responde a extensão bastante lenta do grande artelho (itálico no original) acompanhada, nos casos mais evidentes, da abdução dos outros. A substituiçăo do reflexo flexor normal por este, em extensão, constitui o sinal de Babinski". DeJong dedicou espaço considerável da discussăo sobre as "respotas do feixe piramidal" ao sinal de Babinski ou respota plantar extensora"12. Segundo ele, "estimulação da superfície plantar (...) se acompanha da dorsiflexão dos artelhos, especialmente do grande, simultânea à separação ou abertura em leque dos demais. Estas duas manifestações essenciais foram descritas por Babinski separadamente(...)". Na conhecida monografia organizada pela equipe da clínica Mayo ${ }^{20}$, os autores sustentaram que "o sinal de Babinski consiste na extensāo do grande artelho comumente associada à abertura em leque (abduçăo e discreta flexão) dos outros. Pode-se observar, também, a abduçăo do grande artelho". A literatura nacional clássica não fugiu ao paradigma da época. Aloysio de Castro", por exemplo, escreveu que "as modificaçōes dos reflexos cutâneos na hemiplegia têm a sua mais patente expressāo no sinal de Babinski, inversão da forma normal do reflexo cutâneo-plantar, a saber, a extensão dorsal dos dedos do pé (ou, mais raramente, abdução dos mesmos) e, sobretudo, extensão do polegar do pé (...)".

Nos domínios da fisiologia experimental, Fulton e Keller ${ }^{14}$ enfatizaram, em monografia sobre o sinal de Babinski e a filogênese da dominância cerebral nos primatas, que "o próprio Babinski sustentou que a extensão do hálux e a abertura dos artelhos menores constitui sinal específico de lesão do feixe piramidal (...)". No resumo da mesma monografia ${ }^{13}$, Fulton assinalou que realizaram "(...) estudo sistemático sobre as condiçôes necessárias à produçāo do reflexo de Babinski (extensão plantar com abertura em leque dos artelhos menores) em macacos. babuínos e chimpanzés". Tais considerações mostram-se inexatas à luz dos originais, uma vez que Babinski inclinava-se, na maior parte das vezes exatamente para o contrário, isto é, a abertura em leque podia ocorrer em pessoas normais, razāo de sua baixa especificidade em relação à extensão do hálux. Além disso, jamais empregou a palavra lesāo ("injury") para se referir a correlatos anatômicos ou funcionais do sinal, preferindo o termo deliberadamente genérico "perturbação", pois ele mesmo havia atentado para a resposta extensora em condiçōes puramente funcionais - e, portanto, transitórias -, como nos estados pós-comiciais epiléticos ${ }^{2}$. A tradição experimental coniervou-se, no mínimo, ambígua nos anos subsequentes. Escrevendo sobre as funções motoras do córtex cerebral para uma das obras mais completas de fisiologia médica, Henneman ${ }^{16}$ asseverou que, "após uma lesão piramidal, o grande artelho dorsiflete e os outros abrem em leque. Isto se chama sinal de Babinski"(itálico no original). Conceito igualmente imperfeito foi enunciado por Langworthy ${ }^{17}$ ao afirmar que 0 "sinal de Babinski plenamente desenvolvido (the fully developed Babinski sign) das lesðes cerebrais, no sentido da extensão do grande artelho com abertura em leque dos outros, parece resultar da liberação das respostas de evitamento a nível superior, pela perda total ou parcial dos reflexos de exploração. O evitamento distal, ou dorsiflexăo, limitado à resposta do hálux a estímulos de contato móveis, firmes e profundos, representa 
o verdadeiro sinal de Babinski (the true Babinski sign) e, neste sentido, é mais específico para as lesões piramidais". Infelizmente, Babinski jamais distinguiu uma forma "plenamente desenvolvida" de outra, "verdadeira", do sinal.

Reconhecemos que as passagens acima foram obviamente ponderadas a favor do nosso argumento. Assim, para cada exemplo de ambiguidade ou imprecisão, é provável que se encontrem exemplos simétricos de estudiosos igualmente influentes. No capítulo dedicado às consideraçōes anátomo-clínicas sobre a medula e o tronco encefálico, Machado definiu o sinal de Babinski dos casos de lesão cớrtico-espinhal pela "(...) flexão dorsal do hálux" ${ }^{18}$. Silva e Spina-França investigaram o emprego do frio como estímulo do reflexo plantar e

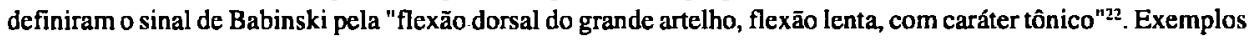
deste tipo, contudo, nāo foram por nós aqui explorados, na busca de transmitir o impacto que se operou em nós mesmos quando, ao deter-nos sobre a questão, verificamos quantas impropriedades rondavam um tema considerado tão bem estabelecido e confiável.

\section{REFERÊNCIAS}

1. Babinski J. Du phénomène des orteils et de sa valeur sémiologique. Sem Méd 1898;18:321-322.

2. Babinski J. Du "phénomène des orteils" dans l'épilepsie. Rev Neurol (Paris) 1899;7:512-513.

3. Babinski J. De l'abduction des orteils. Rev Neurol (Paris), 1903;11:728-729.

4. Babinski J. De l'abduction des orteils (signe de l'éventeil). Rev Neurol (Paris) 1903;11:1205-1206.

5. Babinskj J. Sur le réflexe cutané plantaire (différences dans les réactions corréspondant avec les différences dans le siège de l'excitation). Rev Neurol (Paris) 1907;15:755.

6. Bickerstaff ER. Neurological examination in clinical practice. Ed2. Oxford: Blackwell, 1968:207.

7. Bouchard C, Brissaud É. Traité de médecine. Ed2. Paris: Masson, 1904:63.

8. Castro A. Semiótica nervosa. Ed2. Rio de Janeiro: F. Briguiet \& Cia, 1935:467-468.

9. Chaddock CG. The external malleolar sign. Interstate Med J 18:1026-1038.

10. Cossa P. Physiopathologie du système nerveux: du mécanisme au diagnostic. Ed2. Paris: Masson, 1942:436.

11. Dejerine J. Sémiologie des affections du système nerveux. Paris: Masson, 1914:956.

12. DeJong $R$. The neurological examination. Ed4. Cambridge: Harper \& Row, 1979:456-457.

13 Fulton JF. The Babinski reflex in the monkey, baboon and chimpanzee. J Nerv Ment Dis 1932;75:51-52.

14. Fulton JF, Keller AD. The sign of Babinski in primates: a study of the evolution of cortical dominance in primates. Springfield, I11: Charles C. Tomas, 1932.

15. Gijn J. The Babinski sign and the pyramidal syndrome. J Neurol Neurosurg Psychiatry 1978;41:865-873.

16. Henneman E. Motor functions of the cerebral cortex. In Mountcastle VB (ed). Medical physiology. Ed13. Saint Louis: C.V. Mosby, 1974:774.

17. Langworthy OR. The sensory control of posture and movement: a review of the studies of Derek-Denny Brown. Baltimore: Williams \& Wilkins, 1970:122.

18. Machado A. Neuroanatonia funcional. Rio de Janeiro: Atheneu, 1979:169.

19. Marie $P$, Foix $C$. Les reflèxes d'automatisme médullaire et le phénomène des raccourcisseurs: leur valeur sẻmiologique, leur signification physiologique. Rev Neurol (Paris) 1912;20:657-676.

20. Mayo Clinic and Mayo Fundation. Clinical examination in neurology. Ed6. Saint Louis: Mosby Year Book, 1991:252.

21. Oliveira Souza R, Figueiredo WM. O reflexo cutâneo-plantar em extensão (Babinski, 1896/1898). Arp Neuropsiquiatr 1995;53:318-323.

22. Silva JAG, Spina-França A. Sinal de Babinski: emprego do frio como estímulo sensitivo. Arq Neurpsiatr 1966:24:180-184. 